\title{
Clear widens the field for observations of the Sun with multi-conjugate adaptive optics ${ }^{\star}$
}

\author{
Dirk Schmidt ${ }^{1}$, Nicolas Gorceix ${ }^{2}$, Philip R. Goode ${ }^{2}$, Jose Marino ${ }^{1}$, Thomas Rimmele ${ }^{1}$, \\ Thomas Berkefeld ${ }^{3}$, Friedrich Wöger ${ }^{1}$, Xianyu Zhang ${ }^{4}$, François Rigaut ${ }^{5}$, and Oskar von der Lühe ${ }^{3}$ \\ 1 National Solar Observatory, 3665 Discovery Drive, Boulder, CO 80303, USA \\ e-mail: dschmidt@nso.edu \\ 2 Big Bear Solar Observatory, New Jersey Institute of Technology, 40386 North Shore Lane, Big Bear City, CA 92314, USA \\ 3 Kiepenheuer-Institut für Sonnenphysik, Schöneckstraße 6, 79104 Freiburg, Germany \\ ${ }^{4}$ Large Binocular Telescope Observatory, 933 North Cherry Avenue, Tucson, AZ 85721, USA \\ 5 Research School of Astronomy and Astrophysics, Australian National University, Canberra, ACT 2611, Australia
}

Received 27 October 2016 / Accepted 10 December 2016

\begin{abstract}
The multi-conjugate adaptive optics (MCAO) pathfinder Clear on the New Solar Telescope in Big Bear Lake has provided the first-ever MCAO-corrected observations of the Sun that show a clearly and visibly widened corrected field of view compared to quasisimultaneous observations with classical adaptive optics (CAO) correction. Clear simultaneously uses three deformable mirrors, each conjugated to a different altitude, to compensate for atmospheric turbulence. While the MCAO correction was most effective over an angle that is approximately three times wider than the angle that was corrected by CAO, the full 53" field of view did benefit from MCAO correction. We further demonstrate that ground-layer-only correction is attractive for solar observations as a complementary flavor of adaptive optics for observational programs that require homogenous seeing improvement over a wide field rather than diffraction-limited resolution. We show illustrative images of solar granulation and of a sunspot obtained on different days in July 2016, and present a brief quantitative analysis of the generalized Fried parameters of the images.
\end{abstract}

Key words. instrumentation: adaptive optics - instrumentation: high angular resolution - techniques: high angular resolution methods: observational - Sun: general

\section{Introduction}

To compensate for image degradation induced by turbulence in the Earth's atmosphere, the current facility class adaptive optics (AO) systems at major solar telescopes use one deformable mirror (DM) that is conjugate to the telescope pupil, and one correlating Shack-Hartmann wavefront sensor with a field of view of order 10" (e.g., Rimmele 2004; Berkefeld et al. 2010; Shumko et al. 2014). These classical AO (CAO) systems, which are a single- and pupil-conjugate AO implementation, apply the same wavefront modification equally to all directions in the field of view of the telescope. Optical aberrations due to turbulent airflows, however, can be introduced at any distance from the telescope within the troposphere, and thus can strongly vary with viewing direction. Consequently, the wavefront correction of a DM conjugate to the telescope pupil is usually only valid within a limited viewing angle, which is called the isoplanatic angle (Fried 1982). While CAO systems can provide diffraction-limited images of the Sun within the isoplanatic angle, the image quality may roll off quickly, depending on the instantaneous distribution of the atmospheric turbulence in altitude. The isoplanatic angle is typically of order $5^{\prime \prime}$ to $15^{\prime \prime}$ for observations in the visible light regime at good telescope sites (e.g., Rimmele \& Marino 2011). While adaptive optics has revitalized ground-based solar observations, many problems in solar

\footnotetext{
^ The movies associated to Fig. 1 are available at http://www . aanda.org
}

physics require a much larger diffraction-limited field of view than classical AO systems can provide.

In order to enlarge the field of view that is fully correctable by $\mathrm{AO}$, the third spatial dimension of atmospheric turbulence needs to be incorporated into wavefront correction. Additional DMs can be placed into positions that are optically conjugate to greater distances from the pupil in which strong turbulence occurs. Supplemental wavefront sensors must be deployed to probe the optical aberrations over the targeted field of view in order to steer the DMs. This concept is known as multiconjugate adaptive optics (MCAO; Dicke 1975; Beckers 1987, 1988; Ellerbroek 1994; Rigaut et al. 2000; Fusco et al. 2001; Tokovinin et al. 2001). Stellar observations with MCAO were first carried out by the MCAO Demonstrator MAD at the Unit Telescope 3 of the Very Large Telescope (Marchetti et al. 2007). The MCAO System GeMS of the 8-m Gemini South Telescope (Rigaut et al. 2014; Neichel et al. 2014b) operates in the nearinfrared and constitutes the first, and as yet only, MCAO system routinely used for astronomical observations (e.g., Neichel et al. 2014a). Stellar MCAO systems are being commissioned or planned, for instance, LINC-NIRVANA on the Large Binocular Telescope (Herbst et al. 2016), NFIRAOS on the Thirty Meter Telescope (Herriot et al. 2014), and MAORY on the European Extremely Large Telescope (Diolaiti 2010). Pioneering closedloop experiments with MCAO for solar observations were performed at the Vacuum Tower Telescope (Berkefeld et al. 2010) and at the Dunn Solar Telescope (Rimmele et al. 2010). An advanced solar MCAO system was developed for the GREGOR 
telescope (Berkefeld et al. 2010; Schmidt et al. 2014). Despite the progress that has been made, substantial gain in solar observations with MCAO over those from CAO had yet to be shown. The imminent Daniel K. Inouye Solar Telescope is very likely going to be immediately upgraded with MCAO once demonstrably successful designs for solar science have been implemented (Rimmele et al. 2008). MCAO is considered an integral part of the proposed European Solar Telescope (Soltau et al. 2010). A by-product of MCAO is ground-layer AO (GLAO), which aims to correct for turbulence nearby the telescope only by applying field-averaged correction to the single DM in the pupil (Rigaut 2002). Therefore, the scope of GLAO is to improve the image quality homogeneously across a potentially very large field rather than fully compensating seeing in a small area alone. While success of GLAO is closely related to the instantaneous turbulence distribution in height (e.g., Tokovinin 2004), it is an interesting complementary mode of $\mathrm{AO}$, which can lead to a more efficient use of a telescope if both the observational program and the AO mode are chosen wisely. GLAO might also be attractive for small synoptic solar telescopes, such as SOLIS or SPRING, because of its potential to provide neardiffraction-limited imaging of the full solar disk for this class of telescopes depending on the seeing characteristics of their sites (Rimmele \& Marino 2011). Dedicated solar GLAO experiments without MCAO were performed first at the 76-cm Dunn Solar Telescope (Rimmele et al. 2010) in the visible light regime and recently at the McMath-Pierce Solar Telescope and the Dunn Solar Telescope (Ren et al. 2015) in the near-infrared $H$ band $(1.5-1.8 \mu \mathrm{m})$, but a side-by-side comparison of CAO and GLAO observations demonstrating the merit of GLAO for solar observations was lacking.

We report here the first MCAO observations of the Sun that clearly and visibly widened the corrected field of view compared to quasi-simultaneous observations single DM CAO correction. We also compare these observations with GLAOcorrected images.

\section{Clear, the solar MCAO pathfinder on the NST}

Clear is the MCAO system that we installed on the 1.6-m New Solar Telescope (NST) of the Big Bear Solar Observatory. It provides an integral experimental platform for the development of solar MCAO. Clear was designed for maximal flexibility in order to experimentally evaluate different approaches and concepts (Schmidt et al. 2016). It is built on the experience from the earlier experiments at the VTT and the DST. We use the AO control software KAOS, which was originally developed for the GREGOR telescope (Berkefeld et al. 2012), because we can easily adapt and extend it for our experiments. Clear features three identical 357-actuator DMs, two of which are dedicated to highaltitude seeing, and the third always being located in a pupil image. Because turbulence profiling experiments revealed significant turbulence within the first $7-8 \mathrm{~km}$ above the telescope with relative weightings varying from day to day (Kellerer et al 2012), the two high-altitude DMs can be flexibly conjugated between about 2 and $8 \mathrm{~km}$ on the telescope's line of sight. (The subtropical jet stream at higher altitudes that only occasionally hinders observations in Big Bear is not targeted with Clear at this point.) Multiple correlating Shack-Hartmann wavefront sensor options are available for wide-field sensing in the "star-oriented" MCAO approach (see, e.g., Ragazzoni et al. 1999). A complete overview of the configurations currently available in Clear is listed in Schmidt et al. (2016).

\section{Clear experiments in July 2016}

For the 2016 observing season, we added a new wavefront sensing scheme to Clear that uses a single Shack-Hartmann wavefront sensor unit with either 208 or 112 subapertures, that is, 16 or 12 across the diameter, observing the Sun in the wavelength range $525 \pm 12.5 \mathrm{~nm}$. The field of view in this sensor is $35^{\prime \prime}$. The high-altitude DMs were conjugated to about 3 and $8 \mathrm{~km}$ beyond the telescope on the line of sight, such that they almost continuously cover the first $11 \mathrm{~km}$ (see Rigaut et al. 2000). The wavefront sensor field of view was subdivided into $3 \times 3$ guideregions in which digital image correlation was performed to estimate wavefront slopes in the Shack-Hartmann subapertures; this is the standard method used for solar Shack-Hartmann sensors (Rimmele \& Marino 2011). We used this configuration in a ten-day long observational run at the end of July 2016 when we saw the most impressive boost to the solar image by MCAO so far. We recorded 53" wide field images in the focal plane of Clear with a pco.2000 CCD camera. We took numerous bursts of 450 frames, each $2048 \times 2048$ pixels, with short exposures at 14.7 frames per second, that is, a total time span of approximately $31 \mathrm{~s}$. During the acquisition of a burst, the AO real-time controller KAOS recorded closed-loop data of wavefront sensor measurements and other status data. Typically, we switched the AO operation mode during a burst, meaning that we started with MCAO correction, and after about 150 frames recorded by the pco camera, we instantly switched by a mouse-click to GLAO, and after about another 150 frames to CAO correction. The switching was made by changing the control matrix on-thefly. The simultaneous records of the AO control loop status and the camera bursts allow us to relate the mode of $\mathrm{AO}$ correction to any camera frame. We recorded bursts of 450 continuous frames and switched the mode of correction during the bursts, instead of recording separate dedicated bursts of 150 frames for each mode, in order to avoid downtimes due to data writing during which seeing conditions might change. Hence, we were able to monitor the impact of switching the mode of AO correction on the image plane from one frame to the other.

\section{Effects of CAO, GLAO, and MCAO correction}

We discuss here three illustrative image bursts of solar granulation and a sunspot recorded in July 2016. We have multiple datasets that show similar effects, thus we rule out that effects could be attributed to seeing that might incidentally have changed in the same instant we switched. The images we show were taken on different days at times when the turbulence distribution was such that $\mathrm{CAO}$ correction resulted in the typical small corrected field of order $10^{\prime \prime}$ with a fast roll-off in image quality.

Figure 1 shows the added frames (after dark and flat-field calibration) of the MCAO, GLAO, and CAO-corrected blocks in the exemplary 450-frame bursts. Each picture hence simulates a long-term exposure of MCAO, GLAO, and CAO correction, respectively. (No frame selection was performed, neither did we apply a contrast-enhancing technique such as unsharp masking or modulation transfer function calibration. We did not digitally correct image distortion in the frames before adding.) When we switched to MCAO correction, the widening of the corrected field of view was readily apparent to the naked eye in the live view on the computer screen. Fine structures far from the lock-point of CAO correction became clearly visible. The sunspot example was recorded when the wavefront sensor featured 112 subapertures, and 208 when the granulation examples were taken. In all examples, MCAO correction was 
D. Schmidt et al.: Clear widens the field for observations of the Sun with multi-conjugate adaptive optics
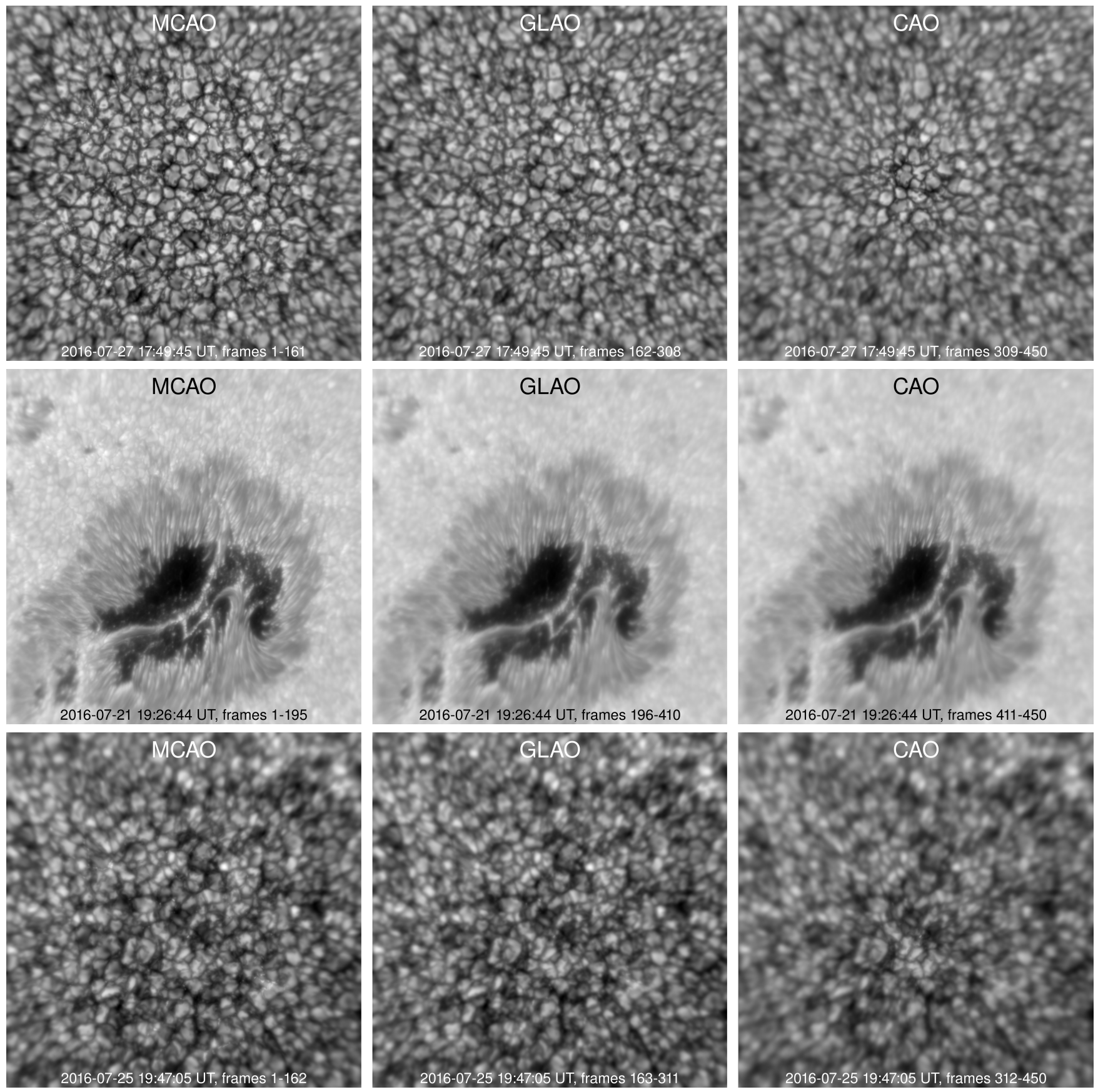

Fig. 1. The Sun observed in a field of view of 53" $\times 53^{\prime \prime}$ with MCAO, GLAO, and CAO correction with Clear on the NST. The top row shows a quiet region of the Sun between 10:49:45 and 10:50:16 PDT on July 27, 2016. The center row shows a sunspot in active region NOAA 12567 on July 21, 2016, between 12:26:44 and 12:27:24. Both bursts were taken with a TiO filter (705.7 $\pm 0.5 \mathrm{~nm})$. The bottom row shows the quiet Sun in $\mathrm{G}$ band $(430.5 \pm 0.25 \mathrm{~nm})$ between 12:47:05 and 12:47:36 on July 25, 2016. Each image shows the sum of the frames within a block with MCAO (left), GLAO (middle), and CAO (right) correction in a continuous burst of 450 frames recorded. Exposure time was $1.6 \mathrm{~ms}$ for the granulation bursts and $11 \mathrm{~ms}$ for the sunspot. Real-time movies are available online.

simultaneously performed with all three DMs, using a total 555 illuminated actuators. We also obtained image bursts with only two DMs correcting (one DM in the pupil and the other DM being one of the high-altitude DMs), which showed inferior performance compared to correction with three DMs.

Figure 2 quantitatively illustrates the effect of the different AO operation modes. We plot the generalized Fried parameter (Cagigal \& Canales 2000) in each MCAO, GLAO, and CAO block of frames. The generalized Fried parameter is an estimate of the Fried parameter (Fried 1965) as it appears after the AO correction. We used the speckle image reconstruction software
KISIP (Wöger et al. 2008) to compute the generalized Fried parameter from the image bursts. KISIP uses the spectral ratio method (von der Lühe 1984) to estimate the generalized Fried parameter from the extended solar scenery.

With MCAO correction, image detail was visible over a wide field, approximately three times as wide as with CAO correction. This factor approximately coincides with the ratio of fields of view used for MCAO and CAO wavefront sensing. The generalized Fried parameter was fairly flat over about $30^{\prime \prime}$ for MCAO correction, whereas CAO correction results in a narrow peak of comparable height. With MCAO correction, detail 

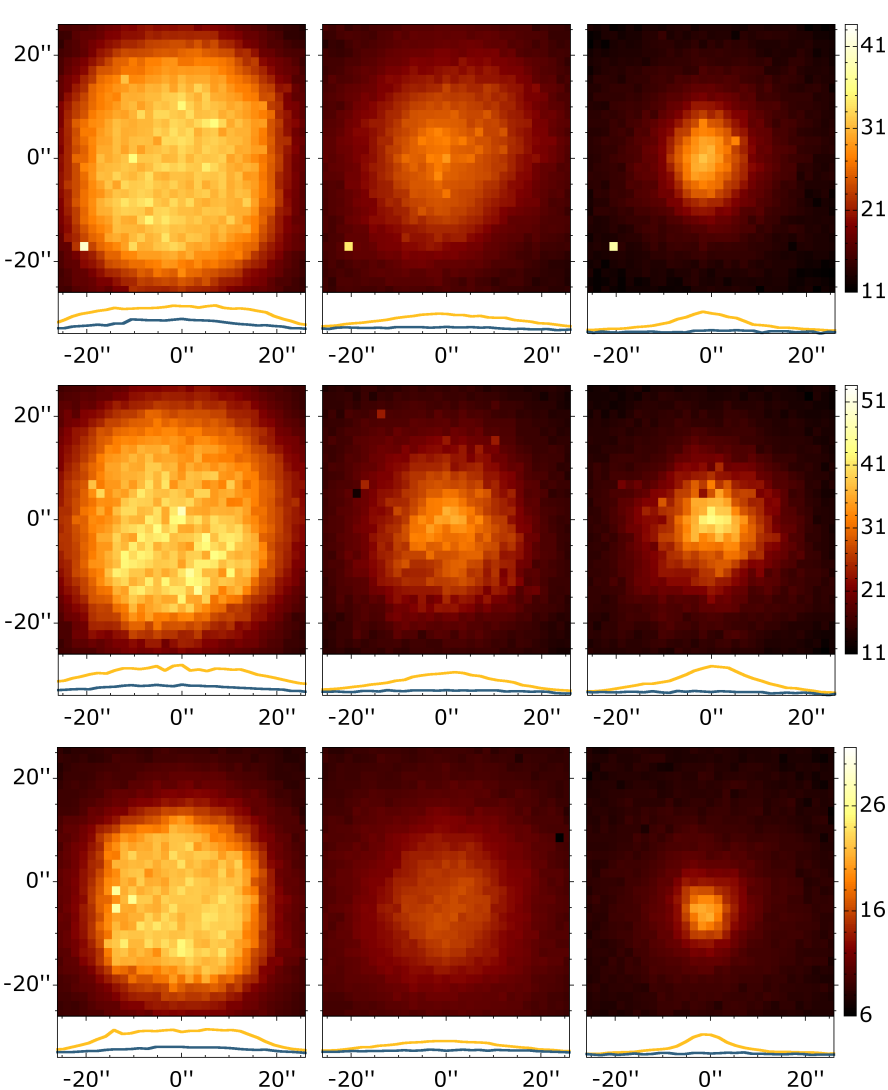

Fig. 2. Generalized Fried parameter in $\mathrm{cm}$ across the field of view in the images shown in Fig. 1. The line plots show the Fried parameter (ranges of the color bars) across the horizontal, where the yellow line is the average of three rows around the maximum of in the CAO plot, and the blue line is the bottom row. (The single bright pixel in the upper plots originates from a dust particle that was not flat-fielded out.)

was still slightly restored even at the margin of the $53^{\prime \prime}$ field image. Ground-layer correction resulted in a lower but more homogeneous image detail over the field compared to classical correction. During our experiments, however, we found that the effect of our GLAO mode of operation was not consistently successful over different situations. We assume that a variable vertical turbulence distribution has a role in this effect.

\section{Conclusions and outlook}

We showed experimentally for the first time and throughout the visible wavelength regime that MCAO can be used to impressively enlarge the corrected field of view for solar observations. This was achieved with three DMs conjugate to 0,3 , and $8 \mathrm{~km}$ and $3 \times 3$ wavefront-sensing guide-regions in $35^{\prime \prime}$ sampled by up to 208 subapertures. Our MCAO pathfinder Clear has proven to be an ideal platform for the development of solar wide-field adaptive optics. We emphasize that we obtained comparable results for observations of granulation, and of a sunspot; these are two typical scenarios prevailing in the photosphere of the Sun. We also showed that GLAO is an attractive complementary AO mode. When the local seeing conditions justify it, we recommend considering an upgrade of existing solar CAO systems with a ground-layer mode. While our latest experiments impressively show the potential of MCAO on solar observations, we will continue to operate and improve Clear as an experimental pathfinder in order to make MCAO a robust tool for widefield high-resolution observations of the Sun. We will likewise continue GLAO experiments. Clear findings will be applied immediately to conception of future wide-field AO systems for the 4-m Daniel K. Inouye Solar Telescope. After further development and experimentation, we plan to make Clear a regularly operating instrument for scientific observations of the Sun on the NST.

Acknowledgements. A number of people contributed in various forms to the Clear project. We name and thank in particular J. Nenow, S. Shoumko, E. Norro, C. Plymate, J. Varsik, R. Coulter (all BBSO), as well as P. Markus and A. Fischer (both KIS). We benefited from technical support of Active Silicon, EDT, Mikrotron, NGC/AOA Xinetics, and Riptide Realtime. We sincerely thank B. Neichel, C. Max, L. Close, and M. van Dam for fruitful discussions at the AO4ELT4 meeting and the 2015 CfAO Fall Retreat that motivated the decrease of the field of view for the wavefront sensor to $35^{\prime \prime}$. We gratefully acknowledge the support of NSF grants AST-ATI-1407597, AGS-1250818 and AFOSR grant FA9550-15-1-0322. NSO is operated by AURA under a cooperative agreement with NSF. The name Clear is an adoption of a name suggested by J. Beckers (then NSO director) in the early phase of what is being built today as the DKIST, which we rediscovered while reviewing the history of telescope names during the July run. D.S. acknowledges P.G. for making mention of it.

\section{References}

Beckers, J. M. 1987, in LEST Technical Report, 28, 55

Beckers, J. M. 1988, Very Large Telescopes and their Instrumentation, in ESO Conf. Workshop Proc., 30, 693

Berkefeld, T., Soltau, D., Schmidt, D., \& von der Lühe, O. 2010, Appl. Opt., 49, G155

Berkefeld, T., Schmidt, D., Soltau, D., von der Lühe, O., \& Heidecke, F. 2012, Astron. Nachr., 333, 863

Cagigal, M. P., \& Canales, V. E. 2000, J. Opt. Soc. Am. A, 17, 903

Dicke, R. H. 1975, ApJ, 198, 605

Diolaiti, E. 2010, The Messenger, 140, 28

Ellerbroek, B. L. 1994, J. Opt. Soc. Am. A, 11, 783

Fried, D. L. 1965, J. Opt. Soc. Am., 55, 1427

Fried, D. L. 1982, J. Opt. Soc. Am. A, (1917-1983), 72, 52

Fusco, T., Conan, J.-M., Rousset, G., Mugnier, L. M., \& Michau, V. 2001, J. Opt. Soc. Am. A, 18, 2527

Herbst, T. M., Arcidiacono, C., Bertram, T., et al. 2016, in Adaptive Optics Systems V, Proc. SPIE, 9909, 99092

Herriot, G., Andersen, D., Atwood, J., et al. 2014, in Adaptive Optics Systems IV, Proc. SPIE, 9148, 914810

Kellerer, A., Gorceix, N., Marino, J., Cao, W., \& Goode, P. R. 2012, A\&A, 542, A2

Marchetti, E., Brast, R., Delabre, B., et al. 2007, The Messenger, 129, 8

Neichel, B., Lu, J. R., Rigaut, F., et al. 2014a, MNRAS, 445, 500

Neichel, B., Rigaut, F., Vidal, F., et al. 2014b, MNRAS, 440, 1002

Ragazzoni, R., Marchetti, E., \& Rigaut, F. 1999, A\&A, 342, L53

Ren, D., Jolissaint, L., Zhang, X., et al. 2015, PASP, 127, 469

Rigaut, F. 2002, in ESO Conf. Workshop Proc., 58, 11

Rigaut, F. J., Ellerbroek, B. L., \& Flicker, R. 2000, in Adaptive Optical Systems Technology, Proc. SPIE, 4007, 1022

Rigaut, F., Neichel, B., Boccas, M., et al. 2014, MNRAS, 437, 2361

Rimmele, T. R. 2004, in Advancements in Adaptive Optics, eds. D. Bonaccini Calia, B. L. Ellerbroek, \& R. Ragazzoni, Proc. SPIE, 5490, 34

Rimmele, T. R., \& Marino, J. 2011, Liv. Rev. Sol. Phys., 8, 2

Rimmele, T., Hegwer, S., Richards, K., \& Woeger, F. 2008, in Advanced Maui Optical and Space Surveillance Technologies Conference, E18

Rimmele, T. R., Woeger, F., Marino, J., et al. 2010, in Adaptive Optics Systems II, Proc. SPIE, 7736, 773631

Schmidt, D., Berkefeld, T., Heidecke, F., et al. 2014, in Adaptive Optics Systems IV, Proc. SPIE, 9148, 91481T

Schmidt, D., Gorceix, N., Marino, J., et al. 2016, in Adaptive Optics Systems V, Proc. SPIE, 9909, 990929

Shumko, S., Gorceix, N., Choi, S., et al. 2014, in Adaptive Optics Systems IV, Proc. SPIE, 9148, 914835

Soltau, D., Berkefeld, T., Sánchez Capuchino, J., et al. 2010, in Adaptive Optics Systems II, Proc. SPIE, 7736, 77360

Tokovinin, A. 2004, PASP, 116, 941

Tokovinin, A., Le Louarn, M., Viard, E., Hubin, N., \& Conan, R. 2001, A\&A, 378,710

von der Lühe, O. 1984, J. Opt. Soc. Am. A, 1, 510

Wöger, F., von der Lühe, O., \& Reardon, K. 2008, A\&A, 488, 375 